\title{
1. Introduction to Big Data Applications in Geography and Planning
}

\section{Mark Birkin, Graham Clarke, Jonathan Corcoran and Robert Stimson}

With growing concern in many countries about the future of large-scale government censuses and surveys (due to increasing costs) increasing interest is being shown in commercial data sets which might generate similar, if not more and better data on national and local economic and social conditions, including the movement and mobility of people and the provision of and access to services and facilities. These public and commercial data sets are often labelled as big data, with much of it being generated through information and communications technologies (ICT), including sensors. Laney (2001) is given credit for giving big data three important characteristics: volume (large quantities of data); velocity (often created continuously); and variety (being structured, semi-structured and unstructured). Since then, Kitchin and McArdle (2016) detail how others have attributed more qualities to big data, including value (adding new insights) and variability (data used in different contexts). Kitchin $(2013,2014)$ provides an excellent review of the characteristics of big data in the social sciences, especially in relation to the properties more associated with small data.

This book on applications of big data - especially its use in spatial analysis and in planning is the result of an academic partnership between geographers at the University of Leeds in the UK and the Universities of Queensland and Melbourne in Australia, facilitated by recent academic leadership of the International Geographical Union's Applied Geography Commission (AGC). Some of the chapters have their origin as presentations to meetings of the AGC.

The editors have all been highly engaged with applied work for many years, and this has been recognised by recent substantial government grants. In the UK, the Economic and Social Research Council (ESRC) launched its Big Data Network in 2014. The Consumer Data Research Centre (CDRC) at the University of Leeds forms a substantial part of this network. The CDRC is directed by a multi-disciplinary group of social scientists from the University of Leeds, University College London (UCL), the University of Liverpool and the University of Oxford. The CDRC at the University of Leeds has been tasked with four goals: to acquire data, to share data, to conduct original research and to develop training and capacity-building. It began with three thematic 'driver projects', in mobility, sustainable consumption and healthy lifestyles. A characteristic of the programme at Leeds from the outset has been its broad multi-disciplinary and multi-institutional character. Core participants span geography, business studies, environmental science, transport and medicine, with other partners in maths, computing, philosophy and law. The CDRC has worked with more than 150 commercial partners to unlock data for its own research and to share more widely with a national community of 
academics and third-party users. The Centre has begun to extend its influence internationally, particularly through applications to obesity and healthy living in the US, Netherlands, New Zealand and elsewhere (Birkin, Wilkins and Morris 2019; Wilkins et al. 2020).

In Australia collaborating researchers have been funded through grants from the Australian Research Council (ARC) over many years for a range of projects involving spatial analysis of social and economic data, transportation and spatial mobility, and the provision of and access to services and infrastructure. In addition, the ARC funded the establishment of the ARC Research Network in Spatially Integrated Social Science (ARCRNSISS) in 2004, and under the Australian Government's National Collaborative Research Strategy (NCRIS) the Australian Urban Research Infrastructure (AURIN) was established in 2010 to build an online data and interrogative analytics facility to support urban and built environment research through collaborative partnership between universities, government agencies and some private sector entities.

This book is an important publication associated with the initiatives such as those described above. Debates on big data have produced many issues around potential advantages and disadvantages. We shall concentrate here on three major issues/debates and argue that the material in the chapters of this book will make a significant contribution to each (for those wanting more in-depth study of pros and cons then see the major texts on big data by Kitchin (2014), Chen and Zhang (2014), Mayer-Schönberger and Cukier (2013), and Marz and Warren (2015). The greatest debate (certainly in geography and planning) has been around the nature of theory building and the analytical techniques needed to process and analyse big data. Kitchin (2013, 2014) again provides a good summary of these arguments.

There is an assumption for many that future research using big data may be largely inductive - 'letting the data speak for themselves', thus leading to the formulation of new models that by definition seemingly 'fit' the data. This suggests new and more powerful techniques will be needed for data mining (Wu et al. 2013). That, however, rings alarm bells for many other academics - a danger that models can be formed with little or no explanatory power (which is especially important when trying to add prediction to static data analysis). Miller and Goodchild (2015) discuss this debate with reference to other periods in the history of quantitative geography.

Whether inductive or deductive methods are used, there is an acknowledgement that big data analysis will require a more ambitious programme of methodological innovation motivated by: (i) a realisation of the need for new methods to deal with big data, for example to handle the lack of uniformity in quality, completeness and coverage; and (ii) growing recognition that data volumes alone are far from a complete replacement for theory and that new methods will be required to infer patterns from real time data streams. Sivarajah et al. (2017) provide a useful discussion on the challenges big data pose to analytical techniques while Singleton and Arribas-Bel (2019) urge geographers not to get left behind and to work with scientists from other disciplines to create a new era of 'geographic data science'.

In response, we hope the collection of papers in this volume show first that deductive theory building and traditional quantitative techniques can still be used to great effect in big data analysis and modelling. These methods include discrete choice modelling, microsimulation and agent-based modelling, spatial interaction modelling, geodemographics, GIS, multi-level modelling and cluster analysis. At the same time a number of the chapters introduce novel data analysis techniques which are being increasingly imported into geography from other disci- 
plines (analogues here perhaps with the history of quantitative techniques used in geography and planning since the 1960s; see Arribas-Bel and Reades 2018).

The quality of big data has been challenged by many, and perhaps proffered by some as an excuse not to engage. Goodchild (2013) and Miller and Goodchild (2015) discuss quality issues in more detail, especially the problems that may arise in meeting the normal scientific standards of replicability and rigorous sampling (see also Cai and Zhu 2015). As Lansley et al. (2019, p. 547) remark:

[I]t is all too easy to focus on the data exploration and pattern discovery, identifying correlations that may well be spurious as a result of the sheer volume of data and the number of events and variables measured. With enough data and enough comparisons, statistically significant findings are inevitable, but that does not necessarily provide any real insight, understanding, or identification of causal relationships.

This suggests we need to think carefully about quality, data cleaning and data being fit for purpose. Related to quality are concerns over representation and ethics. Graham and Shelton (2013, p. 259) summarise their hopes and fears succinctly:

we hope that geographers of all stripes are able to productively and critically employ big data to address long-standing questions of social justice, inequality, and our relationship to the environment, among other concerns. Unfortunately, our hopes are outweighed by fears about the persistent unevenness of representation, limited possibilities for participation, barriers to research and their implications for governance, privacy, and our ways of knowing the world.

Similarly, Lansley et al. (2019) argue that some big data sets present very detailed footprints of user locations making it possible for analysts to distinguish personal characteristics about individuals. Sivarajah et al. (2017) talk more about major challenges around privacy, security and data governance. These are again very legitimate concerns and re-emphasise why care must be taken not to expose individual data and why aggregation is often almost always necessary.

We hope the chapters in the book will (at least in part) help to address the concerns over representation outlined above. Although each chapter discusses the potential of at least one major big data source there is a key recognition throughout the book that for most social science problems, which are relevant to either policy or more theoretical academic studies, it is unlikely to be the case that all the answers may be found in a single novel data source, but rather that a combination of such sources are required. Thus, the variety of data used in the applications in the book should be a source of some consolation here to those still concerned with representation. If one source is heavily skewed towards a particular activity or sub-group, then maybe this can be compensated by another source, which has different characteristics (see also Birkin 2018). Many of the chapters show that it is possible to cross-validate one or more sources of data which are used for analysis against one or more data sources of data which are external to it. However, inevitably, we have to recognise that all data has biases and issues of representation - that is as true for big data as is for small-scale surveys and interviews undertaken more widely in critical human geography. In relation to privacy and ethics it is worth noting that the UK's Digital Strategy has a major strand on unlocking the power of data in the UK economy and improving public confidence in its use. In relation to a number of the chapters in this book, improved public confidence has been achieved at least in part through the secure mechanisms and ethical protocols which CDRC has adopted around sharing of data, 
and more actively through the partnerships and campaigns which are targeted towards public confidence.

It also seems clear that proponents of big data also still need to win over many sceptics about the contribution that the new data can make to traditional social science problems. How can big data supplement or even replace traditional survey data in the future? Although the literature is awash with articles discussing issues around big data there are fewer examples shown of the contribution big data can make across many different areas of geography and planning. Birkin (2018, p. 1) notes that:

academic studies that exploit novel big data sources are pitifully scarce. The primary reason for this is simply that data is typically generated by commercial businesses, or in some cases government organizations, and these data owners have no mandate and little motivation for sharing their assets with academic partners.

Kitchin (2013) makes a similar point in terms of academic access to commercial data sets, although Marr (2016) shows many interesting applications which illustrate how companies themselves are benefiting from using big data.

Within the rest of this book there are 16 applications of big data analytics including case studies around key topics such as demography and migration, retail and consumer analytics, health care planning, urban planning and transport studies. Thus, a major contribution of the book is to show how researchers are accessing big data, what that data looks like and how such data can offer new insights and knowledge in their various disciplines. The book illustrates how data sets leveraged from commercial sources can greatly improve the data we have traditionally worked with in academic geography and planning and hence the insights we have thus far been able to make. This will be illustrated through a variety of international applications, some of which are direct collaborations with government and industry sources. These applications will also discuss data access and, where necessary, data cleaning and preparation for spatial analysis.

The rest of the book is structured as follows. Chapters 2-5 explore applications of big data within population geography, more specifically migration analysis. As is noted at the start of each of these chapters, data on migration has been scarce in most countries with only limited coverage in traditional censuses, where often the only migration question relates to the last previous move (sometimes only within the last 12 months). Thus, it is acknowledged that novel data sources would be particularly useful in this area. In Chapter 2, Alexander explores the potential of social media data in the US to offer fresh insights into migration. She uses Facebook (the Ads Manager) which in the US provides an 'Expats' variable, which indicates whether a person lived in a particular country in the past (but is currently residing somewhere else). There is currently information available for 89 different expat origin groups. This variable can be used to get an estimate of migrant stocks in a particular place. If multiple waves of Facebook data are collected over time, this variable can also be used to infer flows. Then, Lomax (Chapter 3) analyses data obtained from Zoopla, an online estate agency which collects continuous data on house moves across the UK (for buyers and renters). He compares the data to the totals we can get from other migration sources and consequently discusses the pros and cons of this data. In Chapter 4, van Dijk et al. combine a number of large-scale government data sets including electoral registers and data from the UK Land Registry to provide alternative estimates of migration to those found in the UK census. These data sets are continually 
updated so surnames can be followed across the country - they also show how this data can be linked to an 'ethnicity estimator' produced in collaboration with many other projects at UCL in order to also consider migration by ethnicity.

The next three chapters (5-7) introduce big data sets and applications related to health care planning. Many countries do have good health care data available for academic usage, including hospital data and data from primary care providers (such as doctors or GPs). However, data confidentiality rarely allows such data to be accessed at small geographical scales. The good news is that new sources of big data are adding to the portfolio of available data and allowing us to ask new questions concerning small-area health variations. In Chapter 5, Marek et al. access a number of large-scale government data sets in New Zealand containing microdata about people and households from a wide range of authoritative datasets and surveys originating in government agencies and non-government organisations. In particular, they explore the Longitudinal Business Database (LBD) containing microdata about businesses and the Integrated Data Infrastructure (IDI) that focuses on the attributes of people visiting or living in New Zealand in order to determine how current residential location and frequency of change affects local enrolment for health services, service access, and health outcomes. In Chapter 6, Comber et al. explore data from pharmacies which is now available in the UK and provides an incredibly rich data set on local prescription rates and types of medicines prescribed. They use this data to explore more on the geography of mental health in the UK. At this stage their analysis is national and shows exciting spatial variations in the uptake of drugs aimed at relieving mental stress. The potential for future more local scale analysis is huge. In Chapter 7, Pontin explores the potential of smartphone technology to provide new data relating to health and fitness. She explores the Bounts and Strava data sets (apps on smartphones) in particular which record data on exercise and fitness $24 / 7$. Thus, no recall data is required preventing recall bias seen in many surveys. She provides a thorough review of their advantages (and disadvantages) when compared to traditional survey data and data from fitness devices often worn whilst exercising.

The next section of the book focuses on consumer analytics, retailing and geodemographics. In Chapter 8, Dolega uses data from the UK Local Data Company to provide an alternative classification of UK shopping centres. By using data for over 3000 retail centres, the data offers a new level of granularity at both national and local scales and provides a broader understanding of the economic role of commercial spaces, beyond merely retail. In Chapter 9, Hood et al. examine how data from retailers' store loyalty cards can help in many areas of retail location analysis. They illustrate this potential through two case studies. First, they show the use of the loyalty cards to produce better calibrated retail location models and, second, to offer fresh insights into new spatial patterns emerging in retail geography, such as the local usage of e-commerce. In Chapter 10, Tranos and Stich explore the archived webpages from the UK's 'Internet archive', a not-for-profit organisation which provides the most complete archive of webpages in the world. By geolocating these data (the text from the archived webpages being scanned for the inclusion of a valid UK postcode) they are able to build a measure of the volume of web content of local interest and test whether the availability of such web content acts as a pull factor for individuals to connect to the internet.

The fourth set of applications focus on urban planning. First, Stimson and Pettit in Chapter 11 give a tour de force review of big data in relation to contemporary themes in urban geography such as smart, healthy and sustainable cities. They review work from geography, urban 
planning and regional science and offer thoughts on issues around current applications of big data and also suggest some future research priorities. In Chapter 12, Leao et al. use smart-card data from urban transport systems in Sydney, Australia to explore and review the Australian Government's new policy on 30-minute commuting belt policy. This huge volume of daily data adds fresh insight into urban commuting patterns and thus helps in evaluating and refining the urban policies reviewed. In Chapter 13, the focus on reviewing long-term urban policies continues with Stern et al. and their collection of big data through crowdsourcing. The sheer volume of data provided on social media sites relating to key policy can be collected, analysed and used to shape future policy. In particular, they examine the crowdsourcing data in relation to the Israel 100 Strategic Plan. The crowdsourcing theme continues in Chapter 14 where Rae and Nyanzu analyse data obtained from the web site 'FixMyStreet'. This data comes from over 1 million user-generated data points relating to the state of local infrastructure (potholes, graffiti, fly-tipping etc.) and allows them to consider afresh the relationship between the state of local services and neighbourhood affluence. A key theme is do lower income areas suffer more from poor infrastructure and do those communities complain less (and hence potentially get less attention from urban planners) than those in higher income areas?

The final set of three papers explore big data in transport geography. In Chapter 15, Tao et al. provide an excellent review of big data applications in transport policy. They focus on applications in China but their comments reflect the state of the art in this field, and references are also provided to similar studies elsewhere in the world. In Chapter 16 we have a second example of the use of smart-card transit data to help plan services more effectively in large cities. This time Wei et al. look at how transport usage in Brisbane, Australia, is impacted by daily fluctuations in weather. They explore the extent to which local weather conditions impact on transit ridership and how this impact varies by transit mode. Finally, in Chapter 17, Odiari et al. use a spatial microsimulation approach to combine three big data sets relating to train travel in the UK. These include the traditional census, the UK National Travel Survey and LENNON, a national ticketing database relating to individual rail trips in the UK. The authors integrate these three different sources using microsimulation in order to provide new model-based insights and additional decision-support for mobility analysis. The value of the work is shown with regard to increased behavioural insights offered by the combined data, a substantive investigation of the phenomenon of 'rail-heading', and the use of models in 'what if?' scenario planning for a specific infrastructure investment.

We hope the readers will explore the different chapters and get more flesh on the bones of the three substantive themes introduced in this chapter.

\section{REFERENCES}

Arribas-Bel, D. and Reades, J., 2018. Geography and computers: Past, present, and future. Geography Compass, 12(10), e12403.

Birkin, M., 2018. Big data for social science research: Big data (Ubiquity symposium). Ubiquity,(January), $1-7$.

Birkin, M., Wilkins, E. and Morris, M.A., 2019. Creating a long-term future for big data in obesity research. International Journal of Obesity, 43, 2587-2592.

Cai, L. and Zhu, Y., 2015. The challenges of data quality and data quality assessment in the big data era. Data Science Journal, 14, 2.

Chen, C.P. and Zhang, C.Y., 2014. Data-intensive applications, challenges, techniques and technologies: A survey on Big Data. Information Sciences, 275, 314-347. 
Goodchild, M.F., 2013. The quality of big (geo) data. Dialogues in Human Geography, 3(3), 280-284.

Graham, M. and Shelton, T., 2013. Geography and the future of big data, big data and the future of geography. Dialogues in Human Geography, 3(3), 255-261.

Kitchin, R., 2013. Big data and human geography: Opportunities, challenges and risks. Dialogues in Human Geography, 3(3), 262-267.

Kitchin, R., 2014. The Data Revolution: Big Data, Open Data, Data Infrastructures and Their Consequences. Sage, London.

Kitchin, R. and McArdle, G., 2016. What makes Big Data, Big Data? Exploring the ontological characteristics of 26 datasets. Big Data \& Society, Jan-July, 1-10.

Laney, D., 2001. 3D data management: Controlling data volume, velocity and variety. In: Meta Group. Available at: http://blogs.gartner.com/doug-laney/files/2012/01/ ad949-3D-Data-Managemen t-Controlling-Data-VolumeVelocity-and-Variety.pdf.

Lansley, G., de Smith, M., Goodchild, M. and Longley, P., 2019. Big data and geospatial analysis. arXiv preprint arXiv:1902.06672.

Marr, B., 2016. Big Data in Practice: How 45 Successful Companies Used Big Data Analytics to Deliver Extraordinary Results. John Wiley \& Sons, New York.

Marz, N. and Warren, J., 2015. Big Data: Principles and Best Practices of Scalable Real-Time Data Systems. Manning Publications Co, New York.

Mayer-Schönberger, V. and Cukier, K., 2013. Big Data: A Revolution that will Transform how we Live, Work, and Think. Houghton Mifflin Harcourt, New York.

Miller, H.J. and Goodchild, M.F., 2015. Data-driven geography. GeoJournal, 80(4), 449-461.

Singleton, A. and Arribas-Bel, D., 2019. Geographic data science. Geographical Analysis, 0, 1-15.

Sivarajah, U., Kamal, M.M., Irani, Z. and Weerakkody, V., 2017. Critical analysis of Big Data challenges and analytical methods. Journal of Business Research, 70, 263-286.

Wilkins, E., Aravani, A., Downing, A. et al., 2020. Evidence from big data in obesity research: International case studies. International Journal of Obesity, 44, 1028-1040.

Wu, X., Zhu, X., Wu, G.Q. and Ding, W., 2013. Data mining with big data. IEEE Transactions on Knowledge and Data Engineering, 26(1), 97-107. 